\title{
Photophysical properties of 5-hydroxyindole (5HI): Laser flash photolysis study
}

\author{
T MISRA, A K DE, S K PAL, T BHATTACHARYA and T GANGULY* \\ Department of Spectroscopy, Indian Association for the Cultivation of \\ Science, Jadavpur, Kolkata 700 032, India \\ E-mail: sptg@mahendra.iacs.res.in
}

\begin{abstract}
Steady state fluorescence emission and transient absorption spectra of 9fluorenone (9FL) were measured in the presence of 5-hydroxyindole (5HI) in highly polar acetonitrile $(\mathrm{ACN})$ environment at ambient temperature. Cyclic voltammetry measurements demonstrate that ground state $5 \mathrm{HI}$ as a donor could take part in highly exothermic electron transfer (ET) reactions with excited 9FL, which should serve as electron acceptor. From the transient absorption measurements it is inferred that in geminate ion-pair (GIP) (or contact ion pair), formed initially due to photoinduced ET, the decay of this contact ion-pair occurs not only through ion recombination (back electron transfer to ground state of reactants), but through the other processes also such as proton-transfer (hydrogen abstraction) from radical cation to anion and separation of ion-pair producing the free ions. From the computed reorganisation energy parameter $(\lambda)$ and experimentally observed $-\Delta G_{E T}^{0}$ values it is hinted that there is a possibility that highly exothermic forward electron transfer reactions in the singlet state $S_{1}$ occur, within present reacting systems, in Marcus inverted region. Back transfer seems to follow the same path. Investigations with similar other reacting systems are underway.
\end{abstract}

Keywords. Photoinduced electron transfer; H-abstraction; neutral radical; transient absorption; first order and second order decay kinetics.

\section{Introduction}

Investigations on the photophysical properties of indole and its derivatives are gaining increasing interest and the photophysics of indole still remains a very useful subject to reveal the mechanism of long range electron transfer in proteins. Lately our research group is involved in studying the photoinduced electron transfer (PET) reaction using various indoles as electron donors. ${ }^{1}$ These indoles were found to undergo highly exothermic electron transfer (ET) reactions in Marcus inverted region. Thus it is expected that artificial photosynthetic devices could be developed using the indoles as donors. Though several investigations have been carried out on various indoles, ${ }^{2-8}$ almost no attention was given to study the electron donating properties of hydroxyindole. Electrochemical measurements demonstrate that half-wave oxidation potential $\left(E_{1 / 2}^{O X}\right)$ of 5 -hydroxyindole $(5 \mathrm{HI})$ is relatively lower $(+0.34 \mathrm{~V})$ than the corresponding value for 5 -methylindole $(5 \mathrm{MI})(+1.50 \mathrm{~V})$. Thus the $5 \mathrm{HI}$ seems to be much potential electron donor than 5MI. The half-wave reduction potential $\left(E_{1 / 2}^{R E D}\right)$ of $9 \mathrm{FL}$ was found to be $-1 \cdot 28 \mathrm{~V}$. Both steady state and time resolved measurements were made on 5HI using the well-

*For correspondence 
known electron acceptor 9FL as its reacting partner. In this paper the results are described.

\section{Experimental}

\subsection{Purification of chemicals}

All the samples 5HI, 9FL (figure 1), supplied by Aldrich, were purified by vacuum sublimation. The solvent acetonitrile $(\mathrm{ACN})$ was distilled under vacuum according to the standard procedure.

\subsection{Spectroscopic apparatus}

At the ambient temperature $(296 \mathrm{~K})$ steady state electronic absorption and fluorescence emission spectra of dilute solutions $\left(\sim 10^{-4}-10^{-6} \mathrm{~mol} \mathrm{dm}^{-3}\right)$ of the samples were recorded using $1 \mathrm{~cm}$ path length rectangular quartz cells by means of an absorption spectrophotometer (Shimadzu UV-Vis 2101PC) and F-4500 fluorescence spectrophotometer (Hitachi) respectively.

2.2a Picosecond single photon counting techniques: Fluorescence decays of the samples used in the present study were recorded using single photon counting set-up (Millenia (5W) CW $532 \mathrm{~nm}$ pumped Tsunami Ti:sapphire Mode locked Laser with pico option (720-850 nm), SHG and THG as the pump source from Spectra Physics USA) with pulsed width $<2$ ps with $250 \mathrm{~nm}$ excitation. IBH $5000 \mathrm{U}$ Fluorescence spectrophotometer was used for picosecond excitation and detection system with MCP-PMT:R3809U (160-850 nm), Polarizers, NIM timing electronics and PC based MCA with utility software and window based data analysis software (IBH software library). Iterative shift of the fitted function as part of $\chi^{2}$ (error $\pm 6 \mathrm{ps}$, standard deviation $\pm 4 \mathrm{ps}$ ). $\mathrm{N}_{2} / \mathrm{H}_{2}$ filled $100 \mathrm{kHz}$ gated lamp is also available for nanosecond measurement as optional.

2.2b Laser flash photolysis: Laser flash photolysis experiments were done, to measure transient absorption spectra, using the third harmonic of a nanosecond Nd: YAG laser system (355 nm) from Quanta Ray (Spectra Physics, GCR-2, Applied Photophysics) with $8 \mathrm{~ns}$ pulse width, pulse energy $150 \mathrm{~mJ} /$ pulse. The laser was used in a right-angle geometry and a $1 \mathrm{~cm}$ path length cell was used in the investigation. The signals were detected using a $250 \mathrm{~W}$ pulsed xenon lamp, Czerny Tuner monochromator and R-928 PMT. The signals were captured by Hewlett-Packard 54201A digitizing oscilloscope. All the measurements were done on deareated solution made by $30 \mathrm{~min}$ argon purging in the solution. Kinetic analyses were carried out using the software described elsewhere. ${ }^{10}$<smiles>Oc1ccc2[nH]ccc2c1</smiles>

$5 \mathrm{HI}$<smiles>O=C1c2ccccc2-c2ccccc21</smiles>

9FL

Figure 1. Molecular structures of 5HI and 9FL. 
Electrochemical measurements (using standard calomel electrode as the reference electrode and tetraethylammoniumperchlorate (TEAP) as the supporting elctrolyte) were performed with a PAR model 370-4 electrochemistry system. ${ }^{10,11}$

\section{Results and discussion}

\subsection{Singlet quenching in fluid solution at the ambient temperature}

The steady state fluorescence of 9FL (excitation wavelength $\sim 377 \mathrm{~nm}$ ) in highly polar $\mathrm{ACN}$ is found to be efficiently quenched (figure 2), without any change in shape and

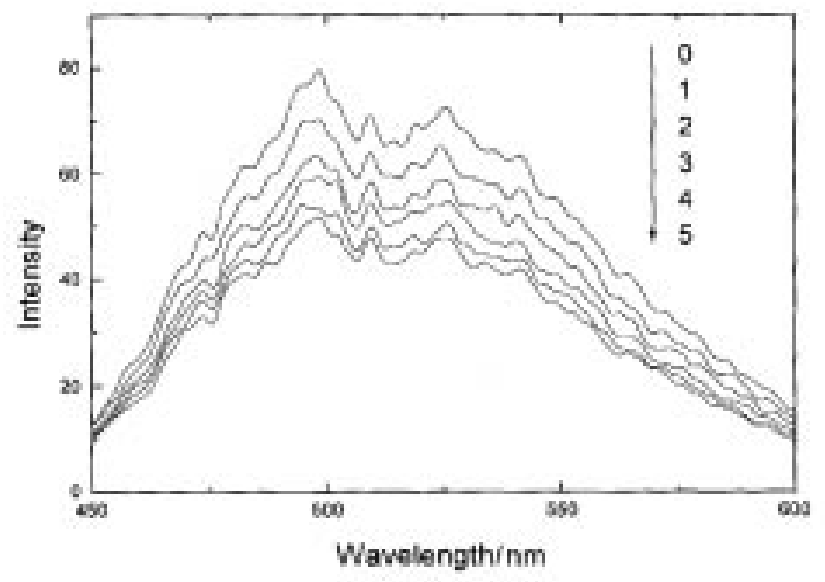

Figure 2. Fluorescence emission spectra of 9FL $\left(\lambda_{\mathrm{ex}}=377 \mathrm{~nm}\right) \quad(\mathrm{C}=7.0 \times$ $10^{-4} \mathrm{~mol} \mathrm{dm}^{-3}$ ) in ACN fluid solution at $296 \mathrm{~K}$ in presence of $5 \mathrm{HI}$. Concentration of $5 \mathrm{HI}\left(\mathrm{mol} \mathrm{dm}^{-3}\right)$ in (0) 0 ; (1) $5.5 \times 10^{-4}$; (2) $1.1 \times 10^{-3}$; (3) $1.6 \times 10^{-3}$; (4) $2.2 \times 10^{-3}$; (5) $3.3 \times 10^{-3}$.

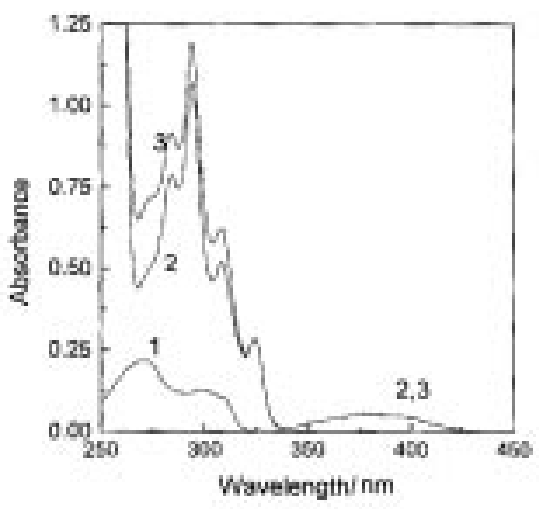

Figure 3. Steady state electronic absorption spectra of $5 \mathrm{HI}\left(\mathrm{C}=1.9 \times 10^{-5} \mathrm{~mol}\right.$ $\mathrm{dm}^{-3}$, curve 1$), 9 \mathrm{FL}\left(\mathrm{C}=3.2 \times 10^{-4} \mathrm{~mol} \mathrm{dm}^{-3}\right.$, curve 2 ) and mixture (curve 3 ) of $5 \mathrm{HI}$ $\left(\mathrm{C}=1.9 \times 10^{-5} \mathrm{~mol} \mathrm{dm}^{-3}\right)$ and $9 \mathrm{FL}\left(\mathrm{C}=3.2 \times 10^{-4} \mathrm{~mol} \mathrm{dm}^{-3}\right)$ in ACN fluid solution at $296 \mathrm{~K} . l=1 \mathrm{~cm}$. 
Table 1. Fluorescence quenching data for the present D-A systems at ambient temperature in ACN solvent, $\tau_{0}$ is the acceptor fluorescence lifetime in absence of the donor (quencher).

\begin{tabular}{lccc}
\hline System & $\lambda_{e m} / \mathrm{nm}$ & $\tau_{0} / \mathrm{ns}( \pm 6 \mathrm{ps})$ & ${ }^{\mathrm{a}} \mathrm{kq} / \mathrm{dm}^{3} \mathrm{~mol}^{-1} \mathrm{~s}^{-1} \times 10^{10}$ \\
\hline $5 \mathrm{HI}+9 \mathrm{FL}^{*}$ & 500 & 14.6 & $1.32(1.58)$ \\
\hline
\end{tabular}

${ }^{a}$ Obtained from steady state fluorescence emission intensity measurements. In this column the values in parentheses are obtained from time resolved measurements *Excited singlet state

energy positions, in the presence of 5HI (quencher). The same observation was made even in the higher concentration of the quencher $\left(\sim 4 \times 10^{-3} \mathrm{~mol} \mathrm{dm}^{-3}\right)$. At the wavelength used for excitation of the fluorescence emission of the acceptor (9FL), the donor molecule is transparent (figure 3). Thus the possibility of occurrence of competitive absorption by the donor as well as its filtering effect on the emission intensity of the acceptor is very slim. In polar ACN fluid solution at the ambient temperature the absorption spectra of the mixture of the donor (5HI) and the acceptor 9FL appear to be just a superposition of the corresponding spectra of the individual donor and acceptor species. This observation supports in favour of the lack of formation of the ground state charge transfer (CT) complex between the donor and the acceptor molecules used.

The bimolecular quenching rate constants, $k_{\mathrm{q}}$, determined from both the linear SternVolmer (SV) plots ${ }^{12,13}$ of (1) fluorescence intensity reduction and (2) fluorescence lifetime quenching of $9 \mathrm{FL}$ as a function of $5 \mathrm{HI}$ concentrations are found to be same within the experimental error (table 1). This observation demonstrates the dynamic behaviour of the observed quenching. It is important to point out here that as the fluorescence quenching of 9FL occurs in the region of 5HI concentrations (figure 2) where the 9FL absorption spectrum is not at all affected, the simple Stern Volmer (SV) equation was used to analyse the quenching phenomena.

The redox potential values in ACN of both $5 \mathrm{HI}\left(E_{1 / 2}^{O X} \approx+0.34 \mathrm{~V}\right)$ and $9 \mathrm{FL}\left(E_{1 / 2}^{R E D} \approx-\right.$ $1.28 \mathrm{~V}$ ), are obtained from the electrochemical measurements by cyclic voltammetry technique. From the observed values it is apparent that 5HI should act as an electron donor in presence of 9FL which would serve as an acceptor in photoinduced ET reactions where the acceptor moiety is excited (the lowest singlet-singlet $(0,0)$ transition energy of the excited chromophore nearly equals to $3.29 \mathrm{eV}$ ). The Gibbs free energy of forward ET reactions $\left(\Delta G_{E T}^{0}\right)$ from well known Rehm-Weller relation ${ }^{12,14,15}$ can be computed for our present reacting systems in highly polar ACN solutions. From the negative value of $\Delta G_{E T}^{0}(\approx-1.67 \mathrm{eV})$ it is apparent that the ET reactions in ACN are highly exothermic $\left(\Delta G_{E T}^{0}<0\right)$ and hence energetically favourable from the thermodynamic point of view. When both the interacting partners are in the ground states, chances of the occurrence of ET reactions seem to be remote, because of the observed positive value of $\Delta G_{E T}^{0}$ $(\sim+1.62 \mathrm{eV})$.

The $k_{\mathrm{q}}$ values (table 1 ) are found to be very close to diffusion-controlled limit ( $k_{\text {diff }}$ for ACN $\sim 2 \times 10^{10} \mathrm{dm}^{3} \mathrm{~mol}^{-1} \mathrm{~s}^{-1}$ ) which indicates that the photoinduced electron transfer (PET) reactions possess a major role in quenching mechanism of the steady state fluorescence emission spectra of 9FL in presence of the electron donor 5HI. Nevertheless for the direct evidence of occurrence of PET, transient absorption measurements were made by using laser flash photolysis technique. The results are described below. 


\subsection{Transient absorption spectroscopy}

In laser flash photolysis experiments pulsed laser excitation at $355 \mathrm{~nm}$, using Nd : YAG laser, was used to excite specifically the acceptor 9FL molecules from the mixture of this acceptor and the donor 5HI.

The transient absorption band of the acceptor 9FL at $420 \mathrm{~nm}$ was observed with $23 \mu \mathrm{s}$ lifetime in ACN solvent. Following observations made by earlier authors ${ }^{16,17}$ this band of 9FL could be assigned to triplet-triplet absorption spectra. Interestingly with addition of the donor $(5 \mathrm{HI})$, in the mixture of 9FL and $\mathrm{ACN}$, a more complex transient spectra were observed with new bands around 460, 500 and $550 \mathrm{~nm}$ in addition to the triplet band at $420 \mathrm{~nm}$ (figure 4). In the same figure the variation of intensity of the transient absorption spectra with changes of delay times between exciting and analysing pulses is shown.

Following the observations made by the earlier authors ${ }^{18-20}$ the new band system at around $460 \mathrm{~nm}$ region has been assigned to the band of 9FL radical anion of contact ionpair (CIP) of the D-A system as the decay at $460 \mathrm{~nm}$ is found to be monoexponential decay from the fitting analysis (figure 5). It is known that radical anions and cations in contact ion-pair recombine by this type of decay kinetics. So in this contact ion-pair, recombination occurs through first order decay. This anionic band is formed due to the photoinduced ET reaction between the excited acceptor 9FL and the present donor in the ground state. The other new absorption band near $550 \mathrm{~nm}$ (figure 4) with half life of $\sim 2 \mu$ s could be assigned to free radical ions (anion) of $9 \mathrm{FL}^{21-23}$ as the decay at $550 \mathrm{~nm}$ obeys second order kinetics (figure 5) where charge recombination between this anion and the donor cation occurs by second order slow diffusion process. These results clearly indicate that the formation of both contact ion-pair and free ions occur by the photoinduced electron transfer mechanism.

In support of the transient absorption spectra where acceptor anion of the contact ionpair (at $460 \mathrm{~nm}$ ) and free or solvent separated anion at $550 \mathrm{~nm}$ regions were observed, we

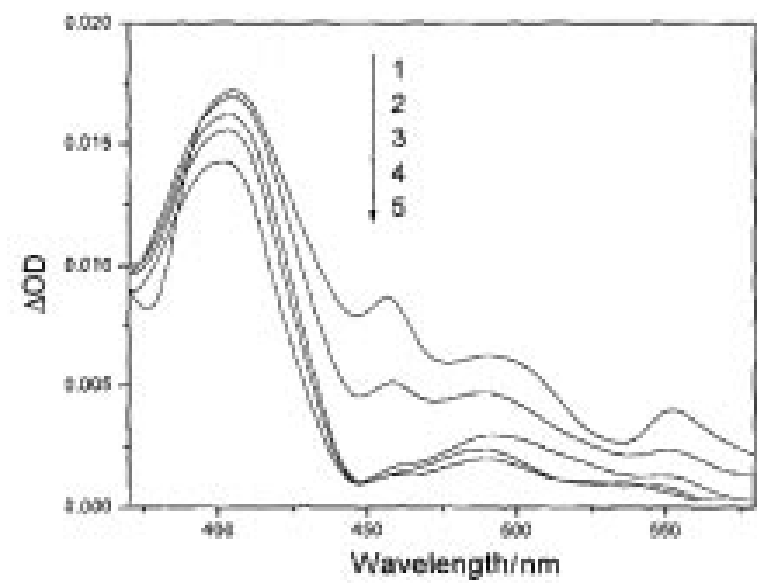

Figure 4. Transient absorption spectra of 9FL (ex. wavelength $\sim 355 \mathrm{~nm}$ using the third harmonic of $\mathrm{Nd}$ : YAG, laser fluence $\sim 150 \mathrm{~mJ} /$ pulse), in ACN (conc. $\sim 5.5 \times$ $10^{-4} \mathrm{~mol} \mathrm{dm}^{-3}$ ) at the ambient temperature in presence of the donor 5HI (conc. $\sim 7.8 \times 10^{-4} \mathrm{~mol} \mathrm{dm}^{-3}$ ) at delay times: $1: 0.5 \mu \mathrm{s} ; 2: 2.0 \mu \mathrm{s} ; 3: 5.0 \mu \mathrm{s} ; 4: 8.0 \mu \mathrm{s}$; 5: $10 \mu \mathrm{s}$. 


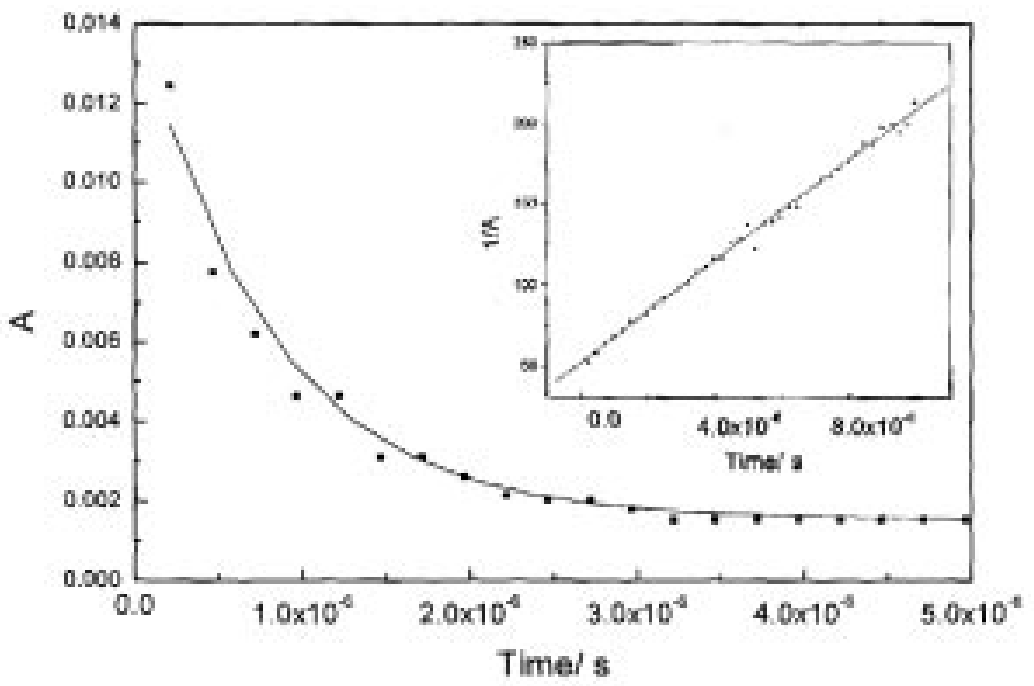

Figure 5. The time profile of the absorbance $(A)$ of the contact ion-pair of 9FL anion radical in presence of $5 \mathrm{HI}$ at $460 \mathrm{~nm}$. The solid line represents the fitting equation: $\mathrm{Y}=A_{0}+A \exp (-t / \tau)$. Inset: plot of $1 / A$ as a function of delay times of free anion of acceptor 9FL at $550 \mathrm{~nm}$ of same system above. The solid line is the second order fitting $\left(r^{2} \sim 0.99\right)$.

measured the steady state electronic absorption spectra of the acceptor 9FL in the presence of metallic sodium in ACN solvent in an attempt to produce artificially 9FL anion according to the reaction mechanism below:

$$
\mathrm{Na}+9 \mathrm{FL} \rightarrow \mathrm{Na}^{+}+9 \mathrm{FL}^{-}
$$

Expectedly in the presence of metallic $\mathrm{Na}$, the absorption bands were found both in the 460 and $550 \mathrm{~nm}$ regions, which gradually disappear in the presence of molecular oxygen. This demonstrates the bands should be due to the formation of anions and the longer wavelength band should be due to the free ion and the shorter one should be responsible for the ion present in the contact ion-pair. It may be pointed out that both for $5 \mathrm{HI}^{+} / 9 \mathrm{FL}^{-}$ and $\mathrm{Na}^{+} / 9 \mathrm{FL}^{-}$ion-pairs, absorption bands at the similar energy positions were found. This observation is due to the fact that in the regions of 460 and $550 \mathrm{~nm}$ only the radical anions $\left(9 \mathrm{FL}^{-}\right)$are accessible experimentally as the absorption of the radical cation of the donor 5HI lies at a much lower wavelength region $(\sim 390 \mathrm{~nm})$ (vide infra).

The decay kinetics at $420 \mathrm{~nm}$ of the transient absorption spectra of 9FL exhibits monoexponential behaviour in the presence of donor (5HI) in polar ACN environment. The decay rate (or the lifetime of the species, $\tau \sim 23 \mu \mathrm{s}$ ) of the $420 \mathrm{~nm}$ band of 9FL remains unchanged even in the presence of the donor 5HI. However, triplet quenching of 9FL by the donor could not be disregarded in this case as the charge recombination lifetime within the present reacting systems, which has been discussed below, was observed to be in the microsecond range. Thus, it may be inferred from the present stage of investigation that electron transfer occurs in this system from ground state donor to both excited singlet 
and triplet of the acceptor but the ET reactions from the triplet is delayed due to its long lifetime.

The time profile of the absorbance of the acceptor 9FL anion (contact ion-pair) in ACN near $460 \mathrm{~nm}$ (figure 5) shows that with further increase of delay beyond $4 \mu \mathrm{s}$, the value of absorbance of the anion remains the same. The lifetime $\tau_{i p}$ is the ion-pair lifetime defined as in $(1,1 \mathrm{a}) .^{24}$

$$
\begin{aligned}
& \tau_{i p}=\left(k_{C R}+k_{d i s}\right)^{-1}, \\
& \phi_{R}=k_{d i s} \tau_{i p},
\end{aligned}
$$

where $k_{C R}$ and $k_{d i s}$ represent the rates associated with geminate recombination and charge dissociation processes respectively.

The yield $\left(\phi_{R}\right)$ of dissociated ion radical formation is given by (1a) and is obtained experimentally by taking the ratio of the absorbance due to the dissociated ions at longer delay time and the initial value estimated by extrapolating the absorbance at $t=0 .{ }^{24}$ Low yield of dissociated ion-radical $\left(\phi_{R} \sim 0 \cdot 1\right)$ was obtained. The rates due to ion-pair recombination $\left(k_{C R}\right)$ and solvent separated ion-pair formation $\left(k_{d i s}\right)$ are computed using (1) and (1a) which are found to be nearly $1.15 \times 10^{6} \mathrm{~s}^{-1}$ and $1.27 \times 10^{5} \mathrm{~s}^{-1}$ respectively for the present D-A systems. Greater value of recombination rate constant indicates in favour of the formation of the contact ion-pair of tight nature.

Monitoring the decay of free anion of $9 F L$ at $550 \mathrm{~nm}$, the ratio of the rate constant of back electron transfer $\left(k_{b}\right)$ to the molar extinction coefficient $(\varepsilon)$ is obtained from the slope of second order plot. ${ }^{19,20}$ The calculated value of $k_{b}$ is equal to $2 \cdot 1 \times 10^{10} \mathrm{M}^{-1} \mathrm{~s}^{-1}$, which is nearly equal to the diffusion controlled limit as expected. This indicates that the decay at $550 \mathrm{~nm}$ results from the slow recombination, by diffusion-assisted process, of the free cationic and anionic species.

The free anion of 9FL is produced by ionic dissociation of contact ion-pair (CIP, scheme 1). The yield, $\phi_{R}$ (discussed above) is actually similar to the $\phi_{\text {sep }}$ (efficiency of free anions formation ${ }^{25}$ ) which can be defined as $\phi_{\text {sep }} \equiv$ [no. of free ions produced]/[no. of contact ion-pair formed]. The rate of charge recombination between contact ions, $k_{b}$, is also computed from (2). This value $\left(1.9 \times 10^{10} \mathrm{M}^{-1} \mathrm{~s}^{-1}\right)$ is very similar to the value obtained from the analysis of the second order fitting (discussed above).

$$
\phi_{\text {sep }}=\left(k_{\text {diff }}-k_{b}\right) /\left(k_{\text {diff }}\right) \text {, }
$$

$k_{\text {diff }}$ is the second order diffusion-controlled rate constant.

The absorption of the counter ion (radical cation of 5HI), appears as a small shoulder near $390 \mathrm{~nm}^{26}$ which is practically hidden over the broad transient absorption band envelope of 9FL (figure 4).

Following the earlier report ${ }^{20}$ the new absorption band found near $490 \mathrm{~nm}$ (figure 4) could be assigned as the neutral radical of 9FL. As from the present study the decay at $490 \mathrm{~nm}$ was found to obey first order kinetics (lifetime $\sim 6 \cdot 2 \mu \mathrm{s}$ ) it shows this band corresponds to contact neutral radical of 9FL.

The shoulder near $510 \mathrm{~nm}$, which looks somewhat prominent at low delay times used and gradually disappears with increase of delay (figure 4) between the exciting and analysing pulses, could be assigned to the neutral radical of 5HI (scheme 1) following the observations made earlier by Zhang et $\mathrm{al}^{27}$ in case of dihydroxy indole molecule. 
These neutral radicals are formed due to cleavage of either $\mathrm{N}-\mathrm{H}$ or $\mathrm{O}-\mathrm{H}$ bond in GIP followed by $\mathrm{H}$-abstraction (scheme 1). This reaction mechanism involves sequential electron-proton transfer, which is an dominant quenching process. From the structural point of view, 5HI have phenolic $\mathrm{O}-\mathrm{H}$ and indolic $\mathrm{N}-\mathrm{H}$ bonds, any one of which can act as hydrogen (proton) donor due to cleavage. ${ }^{27}$ It was observed by earlier workers that if $\mathrm{H}$ of O-H were involved, transient absorption band at around $490 \mathrm{~nm}$ region ${ }^{27}$ and if $\mathrm{H}-$ atom liberates due to cleavage of $\mathrm{N}-\mathrm{H}$ bond, a $510 \mathrm{~nm}$ absorption band appears. ${ }^{27}$ Nevertheless, the broad transient absorption band observed in the present study (figure 4) in this region makes the situation difficult to assign unambiguously the origin of the overlapping bands individually. So at the present stage of investigation no definite conclusions could be reached about the nature of $\mathrm{H}$-atom, whether it belongs to $\mathrm{O}-\mathrm{H}$ or $\mathrm{N}-\mathrm{H}$ group, which undergoes abstraction. Nevertheless, further investigations using the similar donor acceptor systems are now underway to look better insight into the mechanism.

Thus the photoinduced electron transfer seems to be responsible for both the singlet and the triplet quenching reactions. On the basis of the experimental results observed in the present study the reaction mechanisms, as depicted in scheme 1, have been proposed.

The Gibbs free energy changes for back electron transfer reactions by ion pair recombination to form ground state, $\Delta G_{b}(G)$, and excited triplet, $\Delta G_{b}(T)$ of the fluorescer (acceptor) are computed from (3) and (4). ${ }^{28,29}$

$$
\begin{aligned}
& \Delta G_{b}(G)=-E_{1 / 2}^{O X}\left(D / D^{+}\right)+E_{1 / 2}^{R E D}\left(A^{-} / A\right), \\
& \Delta G_{b}(T)=E_{1 / 2}^{R E D}\left(A^{-} / A\right)-E_{1 / 2}^{O X}\left(D / D^{+}\right)+E_{T}^{*},
\end{aligned}
$$

where $E^{*}{ }_{T}$ is the triplet energy level $\left(T_{1} \leftarrow S_{0}\right)$.

In the case of 9FL, Turro $e t a l^{30}$ reported the singlet $\left(S_{0}\right)$-triplet $\left(T_{1}\right)$ energy gap is of about $\sim 2.3 \mathrm{eV}$. Here $\Delta G_{b}(G)$ possess large negative value $(\approx-1.62 \mathrm{eV})$, whereas $\Delta G_{b}(T)$ possess positive value $(\approx+0.68 \mathrm{eV})$ in ACN solvent. Thus from thermodynamical point of view the possibility of formation of ground state by ion recombination is quite high whereas formation of triplet of 9FL by this process could be excluded. But in GIP (or contact ion pair) as depicted in scheme 1, the decay of contact ion-pair occurs not only through ion recombination (back electron transfer to ground state of reactants), but through the other processes also such as proton-transfer (hydrogen abstraction) from radical cation to anion (see above) and separation of ion-pair producing the free ions. Considering the dielectric continuum model, ${ }^{30}$ the total reorganization energy $(\lambda)$ for contact distance $\left(r_{D}\right.$, donor radius $\sim 3.7 \AA$, and $r_{A}$ (acceptor) $\sim 4 \cdot 15 \AA$ ) has been computed $(\approx 1.26 \mathrm{eV})$. This value is less than $-\Delta G_{E T}^{0}$ for forward electron transfer $(E T)(\approx 1.67 \mathrm{eV})$ in the excited singlet state and $-\Delta G_{b}(G)$, for back transfer $(\approx 1.62 \mathrm{eV})$ reactions. These observations are indicative of occurrences of the highly exothermic forward and backward singlet ET reactions in Marcus inverted region. In the lowest excited triplet state of 9FL, lower magnitude of $-\Delta G_{E T}(\sim 0.68 \mathrm{eV})$ relative to $\lambda$ indicate in favour of occurrence of such reactions in normal region. 
$9 \mathrm{FL} * \longrightarrow 9 \mathrm{FL}+h v \quad\left[{ }^{*}\right.$ denotes the lowest excited singlet]

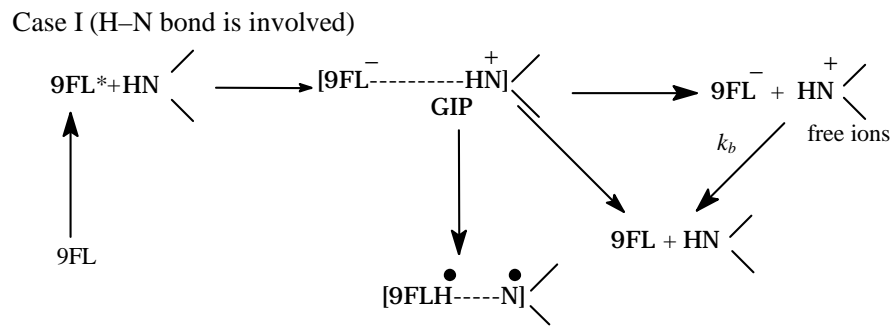

Contact neutral radical

Case II ( $\mathrm{H}-\mathrm{O}$ bond is involved)

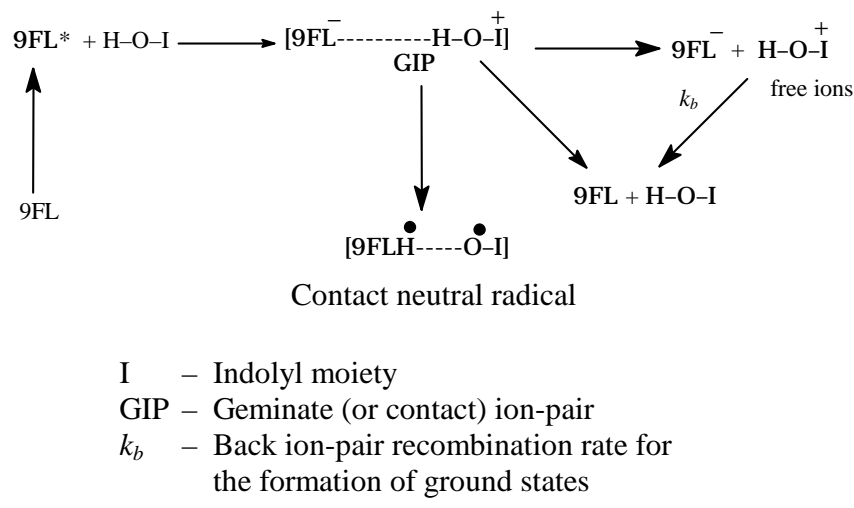

Scheme 1.

\section{Conclusions}

From both electrochemical and time resolved measurements it reveals that the highly exothermic electron transfer reactions occur between the ground state 5HI and excited 9FL $\left(S_{1} / T_{1}\right)$ followed by $\mathrm{H}$-atom abstraction. Long charge recombination lifetime, of the order of microseconds, demonstrates the possibilities of involvements of both the lowest excited singlet and triplet states in quenching reactions through Photoinduced electron transfer processes. These observations indicate that $5 \mathrm{HI}$ is not only a potential electron donor but due to its excellent H-bonding ability, it may use as an antioxidant.

\section{Acknowledgments}

The authors gratefully acknowledge National Centre for Ultrafast Processes, University of Madras, Guindy Campus, Chennai, India for providing facilities for use of laser flash photolysis equipment. We are grateful to $\mathrm{Dr} \mathrm{H} \mathrm{Pal}$, Radiation Chemistry and Chemical Dynamics Division of Bhabha Atomic Research Centre, Mumbai, India for very useful discussions. Thanks are due to our Inorganic Chemistry Department for providing us the facilities of electrochemical measurements. Thanks are also due to Manisankar Maiti of our research group for helping in some experiments. We gratefully acknowledge the 
financial assistances provided by the Council of Scientific and Industrial Research (CSIR), New Delhi in the form of grants and fellowships.

\section{References}

1. Sinha S, De R and Ganguly T 1998 Spectrochim. Acta A50 145

2. Arena A, Martino G, Mezzasalama A M, Mondio G and Saitha G 1991 J. Lumin. 48/49 363, and references therein

3. (a) Share P, Pereira M, Sarisky M, Repinlc S and Hochstrasser R M 1991 J. Lumin. 48/49 204;

(b) Yu H, Colucci W J, Mclaughein M L and Barkley M D 1992 J. Am. Chem. Soc. 114 8449;

(c) Suzuki S, Fujii T, Imai A and Akatiori H 1977 J. Phys. Chem. 811592

4. Sarpel R S, Belletete M and Durocher G 1995 J. Photochem. Photobiol. A88 153

5. Montejano H A, Cosa J J, Garrera H A and Previtali C M 1995 J. Photochem. Photobiol. A86 115

6. Shen X, Lind J and Merenyi G 1987 J. Phys. Chem. 914403

7. Meech S R, Phillips D and Lee A G 1983 Chem. Phys. 80317

8. Hershberger M V, Lumry R and Verrall R 1981 J. Photochem. Photobiol. 33609

9. Vogel A I 1956 Textbook of practical organic chemistry (London: Longmans)

10. Misra T, Ganguly T, Kamila S, Basu C and De A 2001 Spectrochim. Acta A57 2795

11. De A K and Ganguly T 2001 J. Lumin. 92 255, and references therein

12. Maiti M, Sinha S, Deb C, De A and Ganguly T 1999 J. Lumin. 82259

13. Stern O and Volmer M 1919 Phys. Z. 20183

14. Rehm D and Weller A 1970 Isr. J. Chem. 8259

15. Rehm D and Weller A 1969 Ber. Bunsen-Ges. Phys. Chem. 73837

16. Nakajima A 1976 Mol. Photochem. 7251

17. Greeme B I, Hochstrasser R M and Weisman R B 1979 J. Chem. Phys. 701247

18. Kawai A, Yamamoto T, Okutsu T and Obi K 1999 Bull. Chem. Soc. Jpn. 722625

19. Onodera H, Araki Y, Fujitsuka M, Onodera S and Ito O 2001 J. Phys. Chem. A105 7341

20. Hayon E, Ibata T, Lichtin N N and Simic M 1972 J. Phys. Chem. 762072

21. Gersdorf J and Mattay J 1985 J. Photochem. 28405

22. Ito O 1997 Res. Chem. Intermed. 23389

23. Manoj N and Gopidas K R 1999 J. Photochem. Photobiol. A127 31

24. Ganguly T, Sharma D K, Gauthier S, Gravel D and Durocher G 1992 J. Phys. Chem. A96 3757

25. Shimada E, Nagano M, Iwahashi M, Mori Y, Sakaguchi Y and Hayashi H 2001 J. Phys. Chem. A105 2997

26. Grossweiner L I and Usui Y 1970 Photochem. Photobiol. 1153

27. Zhang X, Erb C, Flammer J and Nau W M 2000 Photochem. Photobiol. 71524

28. Encinas M V, Previtali C M and Bertolotti S 1996 J. Chem. Soc., Faraday Trans. 9217

29. Zanini G P, Montejano H A and Previtali C M 2000 J. Photochem. Photobiol. A132 161

30. Kavarnos G J and Turro N J 1986 Chem. Rev. 86401 